Recepción: 20 / 01 / 2017

Aceptación: 20 / 02 / 2017

Publicación: 15 / 03 / 2017

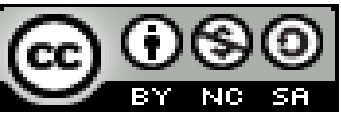

Ciencias Médicas

Artículo Científico

\title{
Factores de riesgos asociados al estado nutricional de personal administrativo
}

\author{
Risk factors associated with nutritional status of administrative staff
}

Os fatores de risco associados ao estado nutricional de pessoal administrativo

Yazmin de las Mercedes Granda Barba ${ }^{\text {I }}$

yazmin.grandab@ug.edu.ec

Amalin M. Chávez Gallegos II

amalin25@ hotmail.com

Elvia P. Aspiazu Miranda ${ }^{\text {III }}$

elvia.aspiazum@ug.edu.ec

Correspondencia: amalin25@hotmail.com

I. Magister en Nutrición; Diplomado en Docencia Superior; Obstetriz; Universidad de Guayaquil, Guayaquil, Ecuador.

II. Diploma Superior en Enfermedades Inmunodeficientes en VIH-Sida; Diploma Superior en Gestión de Desarrollo de los Servicios de Salud; Doctora en Medicina y Cirugía; Universidad de Guayaquil, Guayaquil, Ecuador.

III. Magister en Biotecnología; Especialista en Biotecnología (Opción Biología Molecular e Ingeniería Genética); Bióloga; Universidad de Guayaquil, Guayaquil, Ecuador. 
Yazmin de las Mercedes Granda-Barba; Amalin M. Chávez-Gallegos; Elvia P. Aspiazu-Miranda

\section{Resumen}

Un estudio realizado por la Organización Panamericana de salud (OPS) a través de Iniciativa de Diabetes para la Américas (DIA) y la Federación Internacional de la Diabetes (FID), estimaron que habrían 18 millones de latinoamericanos afectados hasta 2010 llegará a 40 millones para el 2025 con un $69 \%$ en adultos en países en vías de desarrollo y $100 \%$ para los países desarrollados. Las enfermedades metabólicas son causas de morbilidad y mortalidad en el mundo El objetivo: de este estudio es determinar los factores de riesgo en el estado nutricional del personal administrativo de la Universidad Estatal de Milagro. Metodología: Es una investigación de carácter observacional no experimental, descriptivo mediante encuestas y medidas antropométricas. Resultados: Se evalúo 100 trabajadores administrativos de 25 a 60 años, se observó mayor asociación de factores de riesgo en el grupo etario 25 a 45 años que representa el $67 \%$ de los encuestados con afectación del estado nutricional. Conclusiones: El sobrepeso, la obesidad, el sedentarismo y los malos hábitos alimenticios repercutan negativamente en el estado nutricional, generando a largo plazo el desarrollo de enfermedades metabólicas: diabetes, enfermedades cardiovasculares, hipertensión; con una mayor incidencia de obesidad en el grupo etario del sexo femenino de 25 a 45 años.

Palabras clave: Factores de riesgos; estado nutricional; morbilidad; mortalidad. 


\section{Abstract}

A study by the Pan American Health Organization (PAHO) through the Diabetes Initiative for the Americas (DIA) and the International Diabetes Federation (IDF) estimated that 18 million Latin Americans affected by 2010 will reach 40 Million by 2025 with $69 \%$ in adults in developing countries and $100 \%$ in developed countries. Metabolic diseases are causes of morbidity and mortality in the world. The objective: of this study is to determine the risk factors in the nutritional status of the administrative staff of the State University of Miracle. Methodology: This is a nonexperimental observational, descriptive research using surveys and anthropometric measurements. Results: A total of 100 administrative workers aged 25 to 60 years were evaluated. The highest association of risk factors was observed in the age range between 25 to 45 years, representing $67 \%$ of the respondents with affectation of nutritional status. Conclusions: Overweight, obesity, sedentary lifestyle and poor dietary habits negatively affect the nutritional status, generating in the long term the development of metabolic diseases: diabetes, cardiovascular diseases, hypertension; With a higher incidence of obesity in the female age group of 25 to 45 years.

Key words: Risk Factors; nutritional status; morbidity; mortality. 
Yazmin de las Mercedes Granda-Barba; Amalin M. Chávez-Gallegos; Elvia P. Aspiazu-Miranda

\section{Resumo}

Um estudo da Organização Pan-Americana da Saúde (OPAS) através da Iniciativa Diabetes para as Américas (DIA) e da Federação Internacional de Diabetes (IDF) estimou que 18 milhões de latinoamericanos afetados até 2010 chegarão a 40 milhões até 2025 com $69 \%$ em adultos em Países em desenvolvimento e $100 \%$ nos países desenvolvidos. As doenças metabólicas são causas de morbidade e mortalidade no mundo. O objetivo deste estudo é determinar os fatores de risco no estado nutricional do pessoal administrativo da Universidade Estadual do Milagre. Metodologia: Esta é uma pesquisa não-experimental observacional, descritiva usando pesquisas e medidas antropométricas. Resultados: foram avaliados um total de 100 trabalhadores administrativos de 25 a 60 anos. A maior associação de fatores de risco foi observada na faixa etária entre 25 a 45 anos, representando $67 \%$ dos entrevistados com afectação do estado nutricional. Conclusões: o excesso de peso, a obesidade, o estilo de vida sedentário e os hábitos alimentares fracos afetam negativamente o estado nutricional, gerando a longo prazo o desenvolvimento de doenças metabólicas: diabetes, doenças cardiovasculares, hipertensão; Com maior incidência de obesidade na faixa etária feminina de 25 a 45 anos.

Palavras chave: Fatores de risco; estado nutricional; morbidade; mortalidade. 


\section{Introducción.}

La población en estudio labora en el área administrativa y oscila e 25 a 60 años de edad los que están expuestos adquirir problemas nutricionales por llevar una alimentación no adecuada con alto contenido de grasa junto con la inactividad física y su horario de 8 horas de trabajo en 5 días de la semana condición que obliga a la adopción de conductas dietéticas no apropiadas afectaría su estado nutricional y su calidad de vida que también pueden estar influenciados con otros factores como sociales, culturales, económicos, laborales, que en algún momento pueden desarrollar las enfermedades conocida como no transmisibles como: hipertensión arterial, enfermedades cardiovasculares, sobrepeso, obesidad, diabetes y otras. (Vásquez-Garibay, Ávila-Alonso, Contreras-Ramos, Cuellar-Espinosa, \& Romero-Velarde, 2007)

En la actualidad en pleno siglo XXI, el estado nutricional de los adultos se ve afectado por múltiples factores tanto externos como internos; más aún cuando las personas laboran en un trabajo que no exige gran actividad física causando en los individuos un sedentarismo que potenciado con la alimentación diaria generara gran incidencia en que se establezca cuadros de obesidad como hecho de lleva un estilo de vida que es inapropiado para la salud. (Vásquez-Garibay \& Romero Velarde, 2005)

Es por ello que a nivel mundial mediante programas de salud se busca dejar a un lado el sedentarismo y combatir el proceso de obesidad y sobrepeso en aquellas personas que laboran en puestos de trabajo los mismos que pasan 8 horas de trabajo sin tener mayor actividades, y con dietas alimenticias que no aportan nutrientes necesarios para el organismo; generando con ello problemas de diabetes, hipertensión lo que generara consigo que constantemente tengan que abandonar el puesto de trabajo. 
Yazmin de las Mercedes Granda-Barba; Amalin M. Chávez-Gallegos; Elvia P. Aspiazu-Miranda

(Organización Mundial de la Salud, 2012) Estima “que la esperanza de vida podría aumentar en todo el mundo casi cinco años, si se afrontan los factores que afectan la salud, que son responsables de los 60 millones de defunciones cada año". La propuesta generada por la OMS es que aquellos adultos en las edades comprendidas entre 25 a 60 años efectúen en su vida diaria actividades físicas, como deporte, natación por lo menos 75 minutos a la semana para lograr contrarrestar los efectos del sedentarismo en el cual la gran mayoría se encuentra propensos en gran forma en el sobrepeso y la obesidad como causas de la elevada tasa de mortalidad.

Se evidencia que al llevar una vida con un programa de alimentación inadecuado y poco saludable el personal administrativo tendrá problemas en su salud, tales problemas se pueden enumerar como alto contenido de grasa acumulada en su sistema circulatorio por efecto de la inactividad física, al desarrollar procesos administrativos el personal se encuentra con jornadas prolongadas de 8 horas laborales, una hora de receso de actividades es adicionada para efectuar la hora del almuerzo, todo esto realizado de manera secuencial en cinco días laborables a la semana.

La adopción de conductas correctas en nutrición podría ayudar al personal a mejorar su estilo de vida, dichos cambios en su diario vivir facultaría a los individuos a mejorar su condición física y lograr con ello que el trabajo realizado se ejecute de manera eficiente evitando con ello que se baje el rendimiento laboral, se genere ausentismo del personal o llegando a puntos extremos como la accidentabilidad y el aumento en el rango de patologías así también evitar problemas como hipertensión arterial, enfermedades cardiovasculares, sobrepeso, obesidad, diabetes, marcando estas patología en partes a diferentes factores como en lo social, cultural, laboral y económico. 
La presente investigación tiene como objetivo determinar los factores de riesgo en el estado nutricional del personal administrativo de la Universidad Estatal de Milagro para mejorar el estado nutricional del personal mediante un plan Educativo de Promoción y Prevención.

\section{Materiales y métodos.}

La presente investigación a efectuarse en la Universidad Estatal de Milagro de Educación Superior (UNEMI) entidad que lidera la educación superior Ubicada al sur del Cantón Milagro en el Km 1,5 de la vía al Km 26 ( Virgen de Fátima) cuenta con una población de estudio en el área Administrativa de 100 personas las mismas que oscilan en un rango de edad de 25 a 60 años, estas personas su estilo de vida es 50\% se alimentan en su casa a la hora del receso de actividades de $13 \mathrm{~h}$ a $14 \mathrm{~h}$ mientras que el otro $50 \%$ de personal administrativa efectúa su alimentación en la hora de receso el almuerzo en los comedores cercanos al predio universitario, ambos porcentajes almuerzan y luego retornan las actividades; gran parte de la población a estudiarse se encuentran expuestos a múltiples factores de problemas nutricionales.

El presente trabajo tiene un enfoque cuantitativo, de corte transversal no experimental. El enfoque cuantitativo se basa en la relación que existe en entre referencias competitivas estudiadas previamente haciendo un análisis y contrastes sobre los factores de riesgo que son asociados a la mal nutrición en personas del área laboral administrativo de la institución de educación superior, verificando si la acciones de las variables convenidas son determinantes para mantener un bueno o mal estado nutricional. 
Yazmin de las Mercedes Granda-Barba; Amalin M. Chávez-Gallegos; Elvia P. Aspiazu-Miranda

En la investigación se implementa un proceso descriptivo exploratorio investigativo para determinar las variables y desarrollar la respectiva toma de datos que será implementada con carácter cualitativo y cuantitativo con variables antropogénicas, antropométricas, estado nutricional, enfermedades individuales y demás puntos adicionales determinados como necesarios para la investigación, utilizando el instrumento de hoja de recolección de dados para desarrollar posteriormente un plan de información sobre la nutrición necesaria a implementar en los integrantes de la institución.

La recolección de datos está basada en los análisis de los estadios bibliográficos, lectura científica, toma de medidas y entrevista con los participantes de la población estudiada para ser asociados en una base de datos la cual es analizada mediantes gráficos, tablas y referentes estadísticos dando resultado las aprobaciones o rechazo de pruebas de hipótesis.

\section{Método.}

Los métodos a utilizar son teóricos y empíricos, como método teórico utilizaremos el análisis documental, como método empírico se utilizó la observación directa, el análisis y la correlación entre variables. Las variables analizadas son valoración nutricional antropométrica y los factores de riesgos asociados a trastornos nutricionales, los mismos que son sometidas al método de análisis y síntesis.

Se desarrollará un estudio exploratorio, con enfoque cuantitativo al desarrollar un proceso secuencial y probatorio para la idea concebida como identificación de los factores de riesgo que inciden en el estado nutricional, delimitando las variables determinadas en el estudio referentes a factores como edad, sexo, talla, antropométricos para su posterior análisis con la creación de tablas y 
cuadros respectivos que estén acorde a la realidad del entorno investigado, pudiendo así identificar conexiones existentes entre los datos.

\section{Resultados.}

Las prevalencias de sobrepeso y obesidad fueron significativamente mayores en los varones $(59,1 \%$ y $21,6 \%$, respectivamente) respecto a las mujeres $(35,4 \%$ y $14,6 \%$, respectivamente $)$. La literatura señala resultados variables para la asociación obesidad y género. (Rosas, Lama, Llanos, \& Dunstan, 2014)

Se comprobó que valores de CB de $26 \mathrm{~cm}$ en los hombres y $24 \mathrm{~cm}$ en las mujeres servían como puntos de corte para detectar la malnutrición por defecto, por mostrar los valores más altos de sensibilidad y especificidad, que fluctuaron alrededor del $80 \%$ para las variables IMC, áreas de grasa y músculo del brazo. (Berdasco Gómez \& Romero del Sol, 2014)

Se muestra la prevalencia de los factores de riesgo. De las 96 personas encuestadas con relación al IMC, el 43,8 \% de la población presentó sobrepeso, correspondiendo a 17/29 hombres y 25/67 mujeres. La prevalencia de diabetes fue del $1 \%$ y correspondió a una mujer. El $51 \%$ de la población presentó hipercolesterolemia, de los cuales 19/29 fueron hombres y 30/67 mujeres. Un 40,6 \% de la población presentó hipertrigliceridemia, siendo 17/29 hombres y 22/67 mujeres. (Montejano Lozoya, Ferrer Diego, Gonzalo, Martínez-Alzamora, Sanjuan Quiles, \& Ferrer Ferrándiz, 2014)

En el presente estudio investigativo se determinan los estados nutricionales del personal administrativo de la UNEMI, para identificar el riesgo al cual se encuentran afectos los individuos 
Yazmin de las Mercedes Granda-Barba; Amalin M. Chávez-Gallegos; Elvia P. Aspiazu-Miranda

determinados estos por el grado de obesidad o índice IMC resultante en la toma de datos antropométricos desarrollados con el instrumento de recolección de datos.

\begin{tabular}{|c|c|c|c|c|c|}
\hline \multirow{2}{*}{\multicolumn{6}{|c|}{$\begin{array}{l}\text { VALORES DE NUTRICION EN PEI } \\
\text { ADMINISTRATIVO } \\
\text { RANGO DE EDAD ENTRE } \mathbf{2 5 - 4 5}\end{array}$}} \\
\hline & & & & & \\
\hline & $\mathrm{F}$ & $\mathrm{M}$ & SUB & $\mathrm{Fa}$ & $\mathrm{FaA}$ \\
\hline NORMAL & 21 & 6 & 27 & 0.27 & 0.27 \\
\hline SOBREPESO & 11 & 11 & 22 & 0.22 & 0.49 \\
\hline OBESO & 9 & 9 & 18 & 0.18 & 0.67 \\
\hline OBESO TIPO I & 1 & 7 & 8 & 0.08 & 0.75 \\
\hline \multicolumn{6}{|c|}{$\begin{array}{r}\text { RANGO DE EDAD MAYOR A } 45 \\
\end{array}$} \\
\hline NORMAL & 6 & 2 & 8 & 0.08 & 0.83 \\
\hline SOBREPESO & 8 & 5 & 13 & 0.13 & 0.96 \\
\hline OBESO & 2 & 2 & 4 & 0.04 & 1 \\
\hline OBESO TIPO I & 0 & 0 & 0 & 0.00 & 1 \\
\hline total & 58 & 42 & 100 & 1 & \\
\hline
\end{tabular}

Tabla $N^{\circ}$ 1.- Valores nutricionales vs edad. 
Factores de riesgos asociados al estado nutricional de personal administrativo

\begin{tabular}{lcccr} 
& \multicolumn{1}{c}{$\begin{array}{c}\text { Sexo } \\
\text { * }\end{array}$} & & \multicolumn{2}{c}{ Total general } \\
Estado Nutricional & FEMENINO & MASCULINO & 10 & 27 \\
BAJO PESO / NORMAL & 17 & 32 & 73 \\
\hline SOBRE PESO/OBESIDAD & 41 & 42 & 100
\end{tabular}

\begin{tabular}{llcc}
\hline Estado Nutricional & FEMENINO & \multicolumn{2}{c}{ MASCULINO } \\
BAJO PESO / NORMAL & & 15,66 & 11,34 \\
SOBRE PESO/OBESIDAD & & 42,34 & 30,66 \\
\hline
\end{tabular}

Total general

$\begin{array}{ll}\text { P VALUE } & 0,5408 \\ \text { PRUEBA CHI CUADR } & 0,3740\end{array}$

ho: No existe Relacion entre el Estado Nutricional y el Sexo

H1: Si existe Relacion entre el el Estado Nutricional y el Sexo

NO SE RECHAZA LA HIPOTESIS NULA

\begin{tabular}{lccr}
\hline \multirow{2}{*}{$\begin{array}{c}\text { Sexo } \\
\text { Estado Nutricional }\end{array}$ FEMENINO } & MASCULINO & \multicolumn{2}{c}{ Total general } \\
\hline BAJO PESO / NORMAL & $62,96 \%$ & $37,04 \%$ & $100,00 \%$ \\
\hline SOBRE PESO/OBESIDA & $56,16 \%$ & $43,84 \%$ & $100,00 \%$ \\
\hline \hline Total general & $\mathbf{5 8 , 0 0 \%}$ & $\mathbf{4 2 , 0 0 \%}$ & $\mathbf{1 0 0 , 0 0 \%}$
\end{tabular}

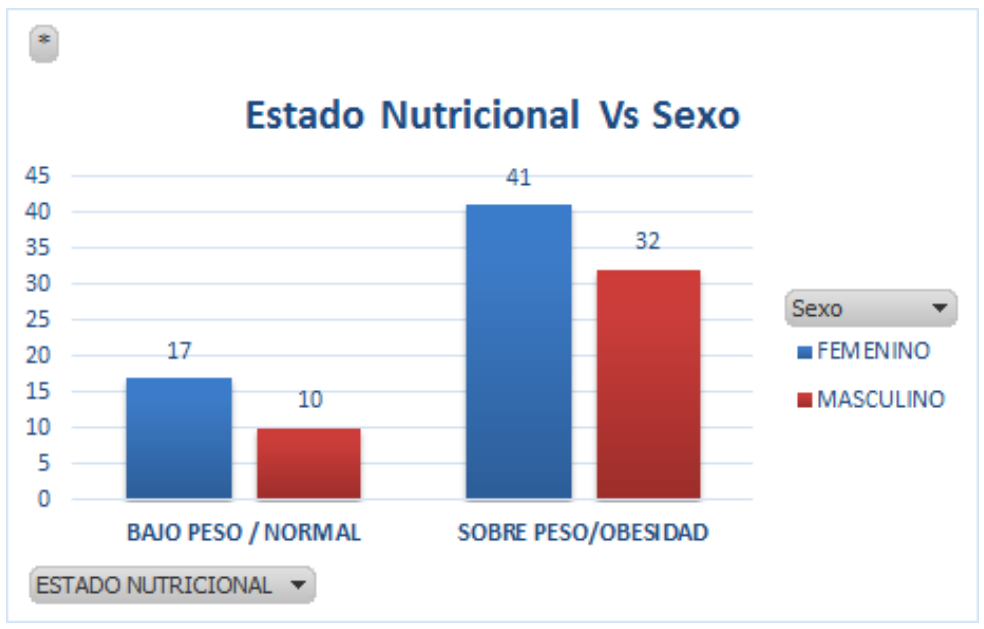

Pol. Con. (Edición núm. 5) Vol. 2, No 3, marzo 2017, pp. 175-196, ISSN: 2550 - 682X 
Yazmin de las Mercedes Granda-Barba; Amalin M. Chávez-Gallegos; Elvia P. Aspiazu-Miranda

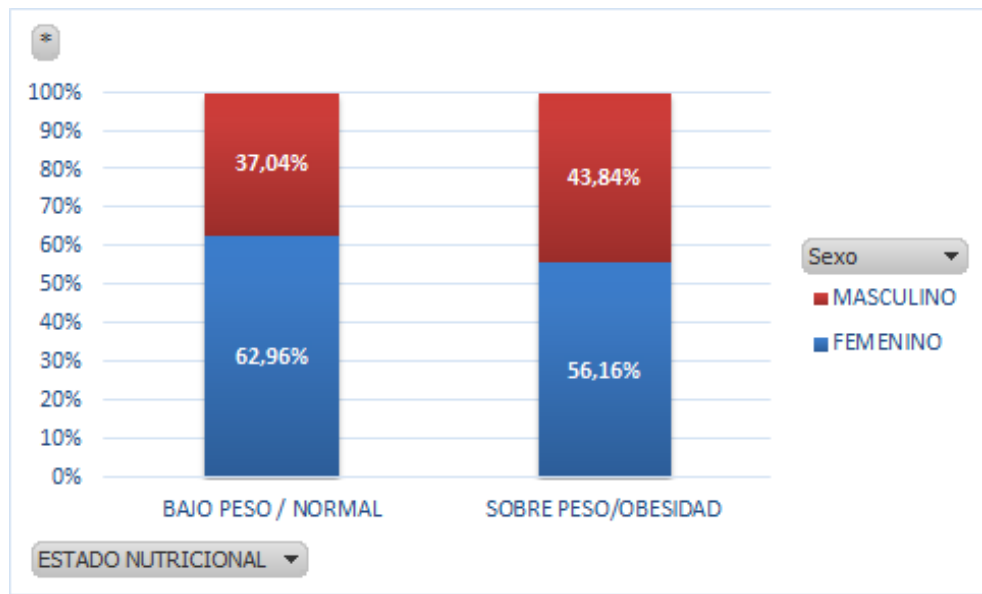

El análisis identifica la aceptación de la hipótesis nula, lo cual el factor de riesgo determinante del sexo no es asociado a la mal nutrición y obesidad de las personas del campo de estudio, sin embargo en ambos sexos existe un porcentaje superior con grados de obesidad con un $56,16 \%$ en mujeres y $43,84 \%$ en hombres.

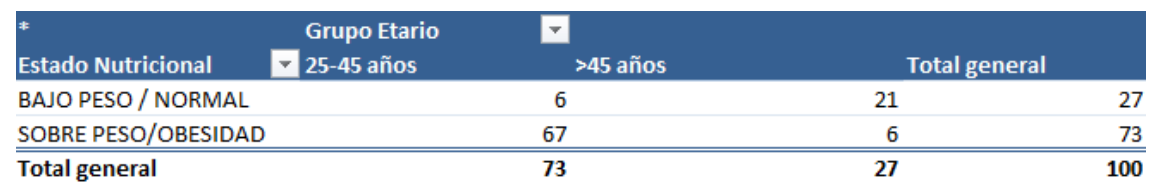

\begin{tabular}{lcr} 
Estado Nutricional & $25-45$ años & \multicolumn{2}{c}{$>45$ años } \\
BAJO PESO / NORMAL & 19,71 & 7,29 \\
SOBRE PESO/OBESIDAD & 53,29 & 19,71 \\
\hline \hline Total general & &
\end{tabular}

P VALUE $\quad 0,0000$

PRUEBA CHI CUADR $\quad 48,3840$

h0: No existe Relacion entre el Estado Nutricional y el Grupo Etario H1: Si existe Relacion entre el Estado Nutricional y el Grupo Etario

SE RECHAZA LA HIPOTESIS NULA

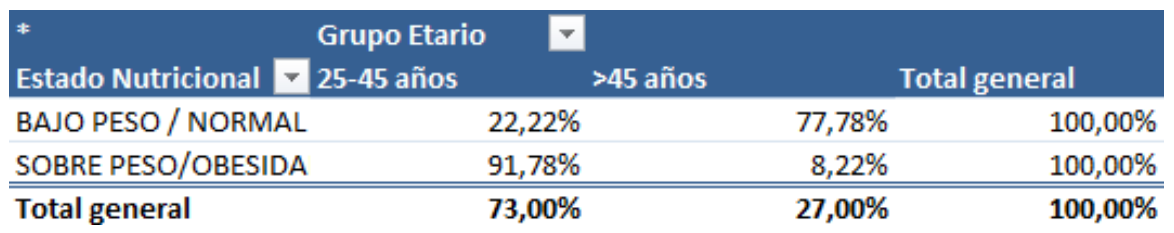




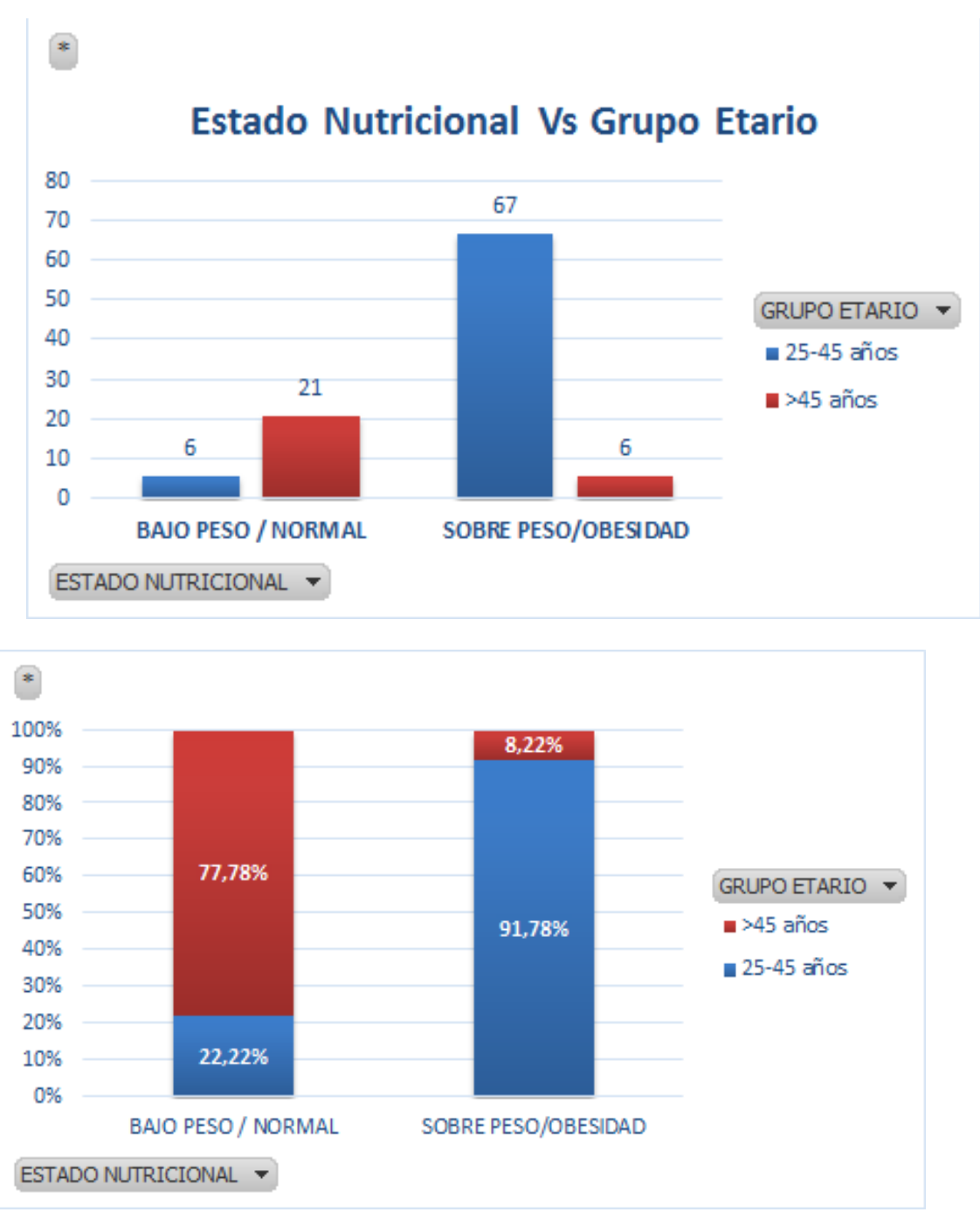

A través del análisis de la información se logra identificar que el nivel normal del peso corporal en los individuos encuestados muestra una depresión delimitada por los rangos de edad que cursan los individuos, pudiéndose apreciar que hasta los 45 años los individuos muestran una tendencia al sobre peso, a partir de los 45 años en adelante existe una mayor probabilidad de mantener un porcentaje alto en el peso adecuado debido a que este factor de riesgo tiene asociación al estado nutricional ya que al llegar a una edad adulta empiezan a cuidar su estilo de vida. 
Yazmin de las Mercedes Granda-Barba; Amalin M. Chávez-Gallegos; Elvia P. Aspiazu-Miranda

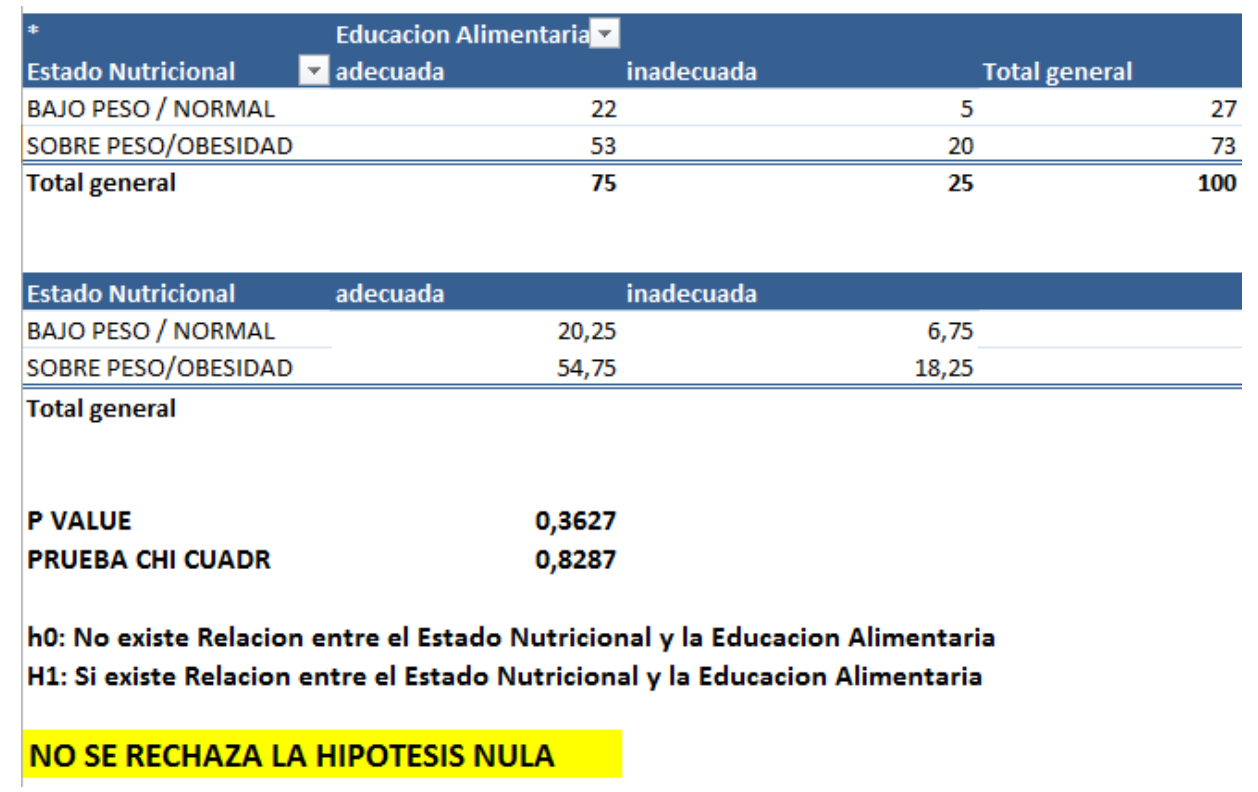

\begin{tabular}{|c|c|c|c|}
\hline * & $\operatorname{limer}=$ & & \\
\hline Estado Nutricional $>$ & & & aral \\
\hline BAJO PESO / NORMAL & $81,48 \%$ & $18,52 \%$ & $100,00 \%$ \\
\hline SOBRE PESO/OBESIDA & $72,60 \%$ & $27,40 \%$ & $100,00 \%$ \\
\hline Total general & $75,00 \%$ & $25,00 \%$ & $100,00 \%$ \\
\hline
\end{tabular}

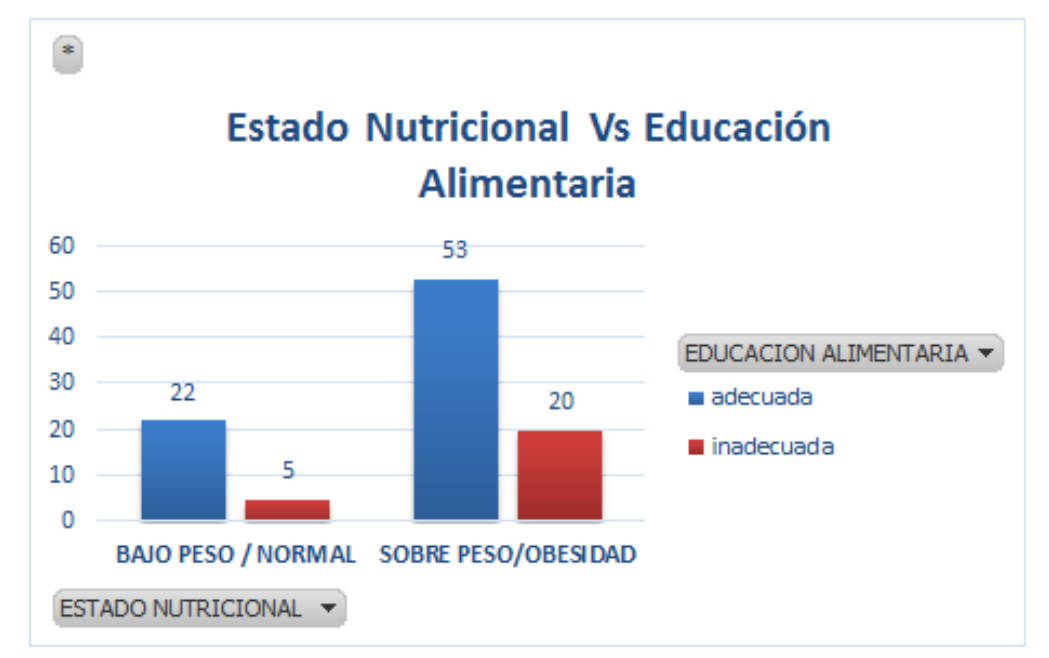

Pol. Con. (Edición núm. 5) Vol. 2, No 3, marzo 2017, pp. 175-196, ISSN: 2550 - 682X 


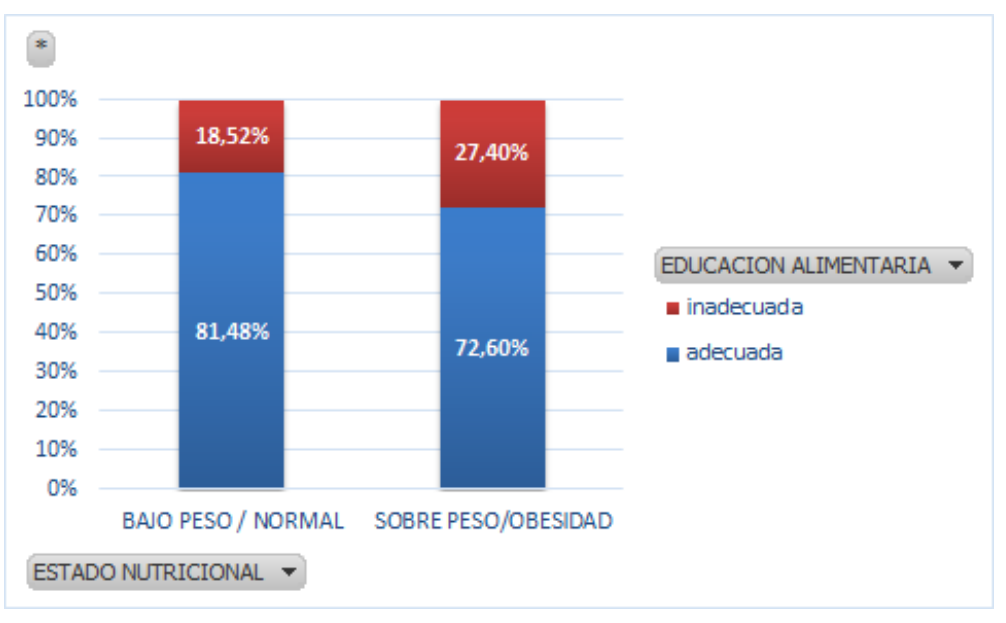

Los valores referentes a las estimaciones de la ingesta de una alimentación adecuada o inadecuada toma cifras muy elevadas al identificar un total del $81,48 \%$ de la población investigada con un peso adecuado y 72,60\% de la población con sobre peso u obesidad considera tener una correcta alimentación, estos valores transgreden en la acción de ser humano ya que al no tener un concepto de alimentos saludables o adecuados e inadecuados toman por su propia cuenta atributos de aceptabilidad sobre los alimentos saludables para el consumo de las personas.

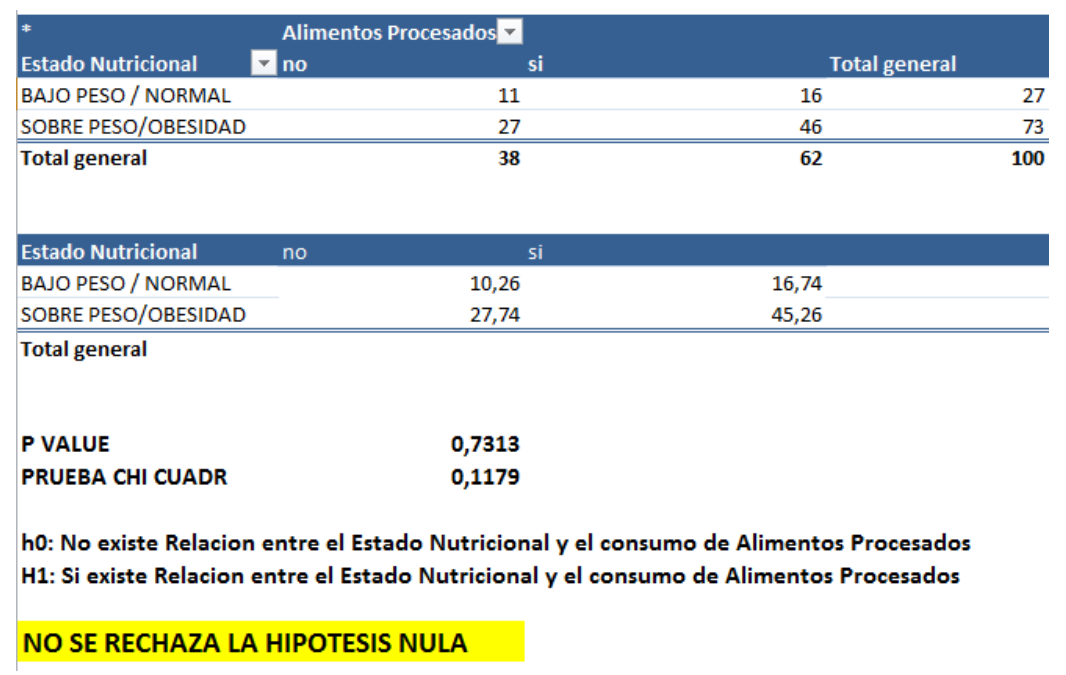

\begin{tabular}{|c|c|c|c|}
\hline \multicolumn{4}{|c|}{$* \quad$ Alimentos Proces -} \\
\hline Estado Nutricional $\nabla$ no & si & \multicolumn{2}{|c|}{ Total general } \\
\hline BAJO PESO / NORMAL & $40,74 \%$ & $59,26 \%$ & $100,00 \%$ \\
\hline SOBRE PESO/OBESIDA & $36,99 \%$ & $63,01 \%$ & $100,00 \%$ \\
\hline Total general & $38,00 \%$ & $62,00 \%$ & $100,00 \%$ \\
\hline
\end{tabular}




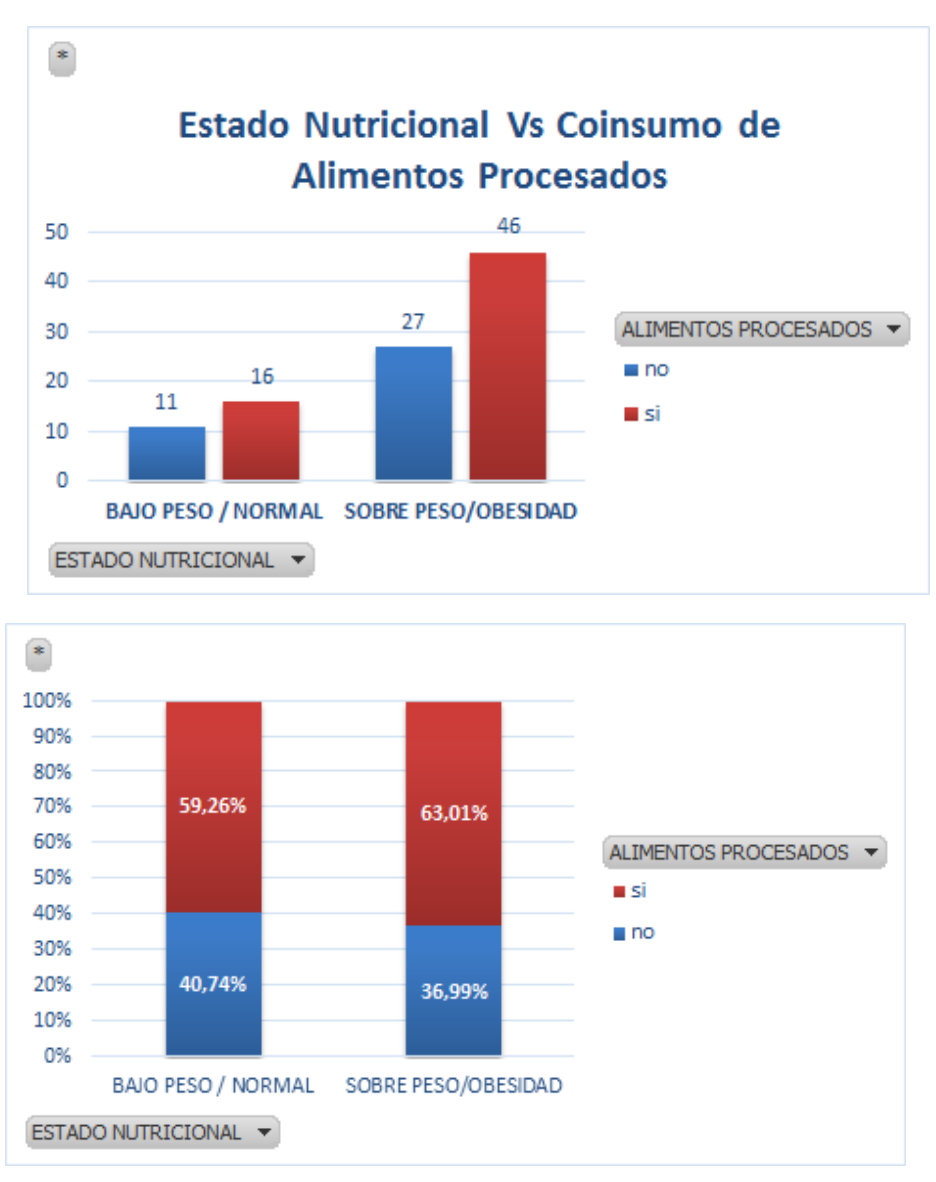

Los valores referentes a la ingesta de alimentos procesados están caracterizados por un gran porcentaje de miembros participantes que se encuentran que hace parte de su dieta los alimentos procesados, teniendo un valor de $59,26 \%$ en personas con un peso adecuado y $63,01 \%$ en personas con sobrepeso u obesidad, este factor de riesgo tiene una gran asociación en la nutrición de las personas ya que existe el desconocimiento sobre las porciones de consumo, contenido nutricional e ingesta de aditivos alimentarios en exceso, todo esto se ve afectado o puede afectar a la salud de los individuos. 
Factores de riesgos asociados al estado nutricional de personal administrativo

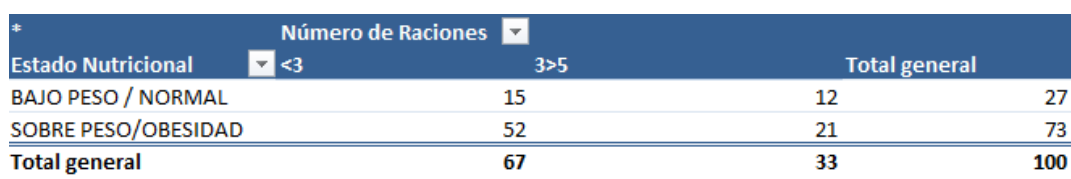

\begin{tabular}{lr} 
Estado Nutricional & $<3$ \\
\hline BAJO PESO / NORMAL & 18,09 \\
\hline SOBRE PESO/OBESIDAD & 48,91 \\
\hline \hline Total general & \\
& \\
& \\
P VALUE & $\mathbf{0 , 1 3 8 8}$ \\
PRUEBA CHI CUADR & $\mathbf{2 , 1 9 1 0}$
\end{tabular}

h0: No existe Relacion entre el Estado Nutricional y el Número de Raciones H1: Si existe Relacion entre el Estado Nutricional y el Número de Raciones

\section{NO SE RECHAZA LA HIPOTESIS NULA}

\begin{tabular}{|c|c|c|c|}
\hline \multicolumn{4}{|c|}{ * $\quad$ Número de Racio $\nabla$} \\
\hline Estado Nutricional $\nabla<3$ & $3>5$ & & \\
\hline BAJO PESO / NORMAL & $55,56 \%$ & $44,44 \%$ & $100,00 \%$ \\
\hline SOBRE PESO/OBESIDA & $71,23 \%$ & $28,77 \%$ & $100,00 \%$ \\
\hline Total general & $67,00 \%$ & $33,00 \%$ & $100,00 \%$ \\
\hline
\end{tabular}

\section{Estado Nutricional Vs Número de Raciones}
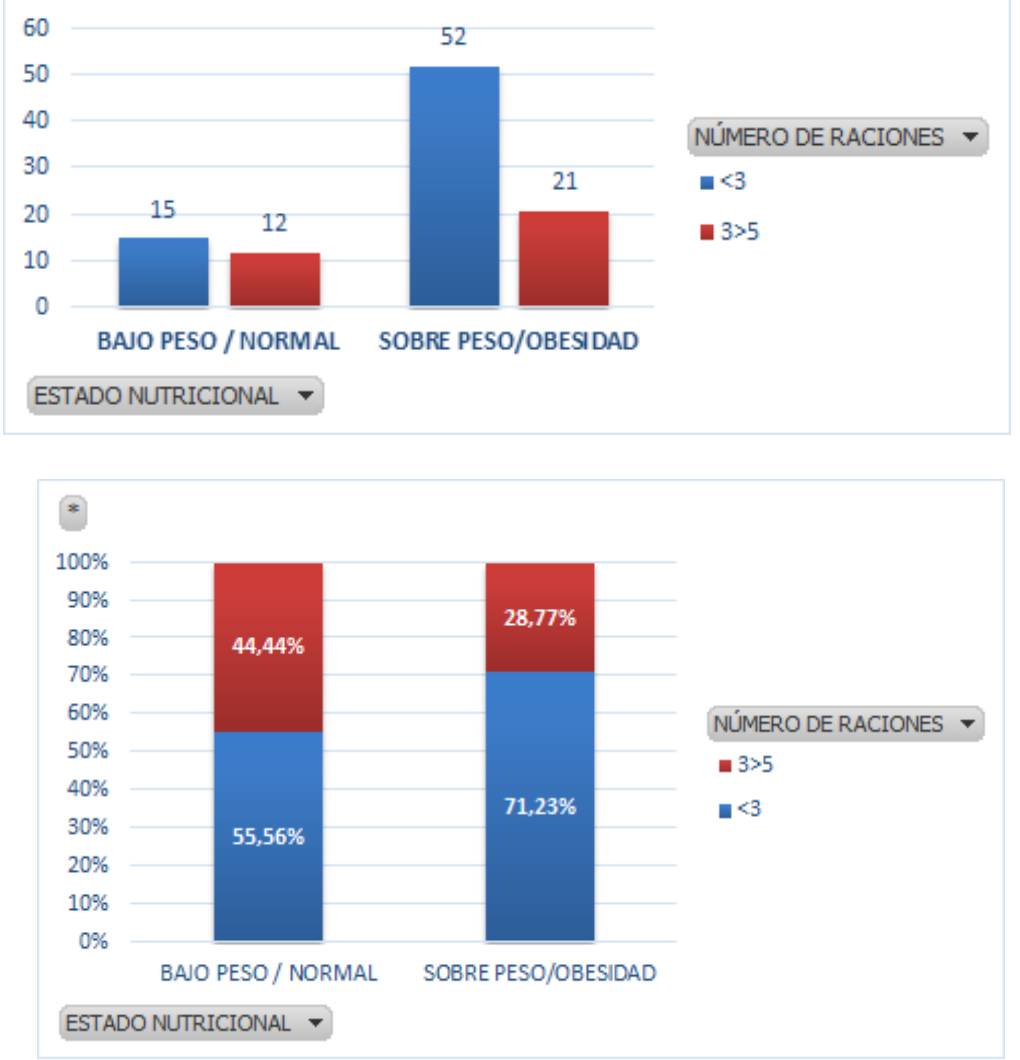
Yazmin de las Mercedes Granda-Barba; Amalin M. Chávez-Gallegos; Elvia P. Aspiazu-Miranda

El factor de afectación sobre las raciones alimentaria de la población estudiada los refleja que $55,56 \%$ y $71,23 \%$ de peso adecuado y sobre peso consume raciones de comidas inferiores o iguales a tres comidas por día, teniendo una malnutrición por la descomprensión sobre los hábitos y raciones alimenticias siendo este un factor que acepta la hipótesis nula, tiene mucha relación a la cantidad y raciones que consume las personas.

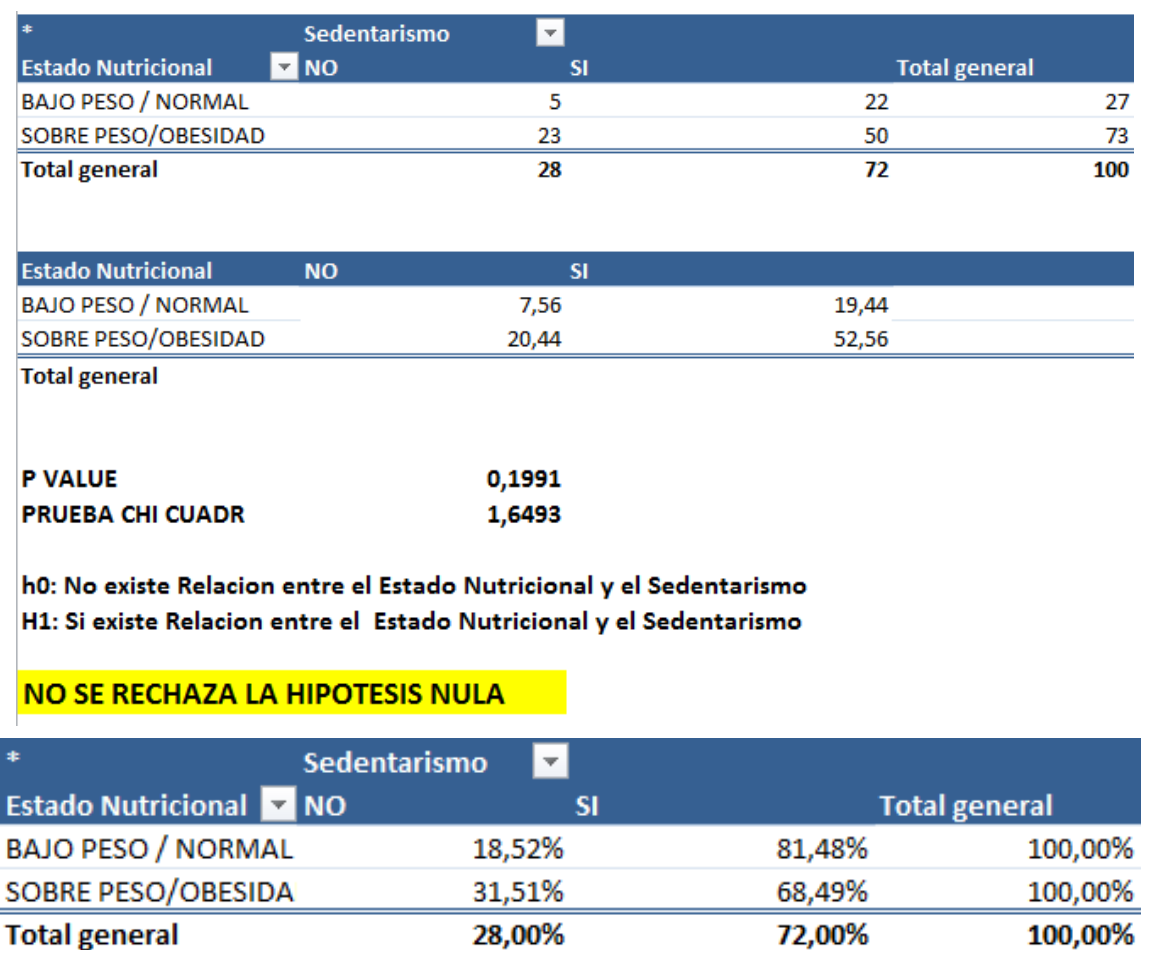



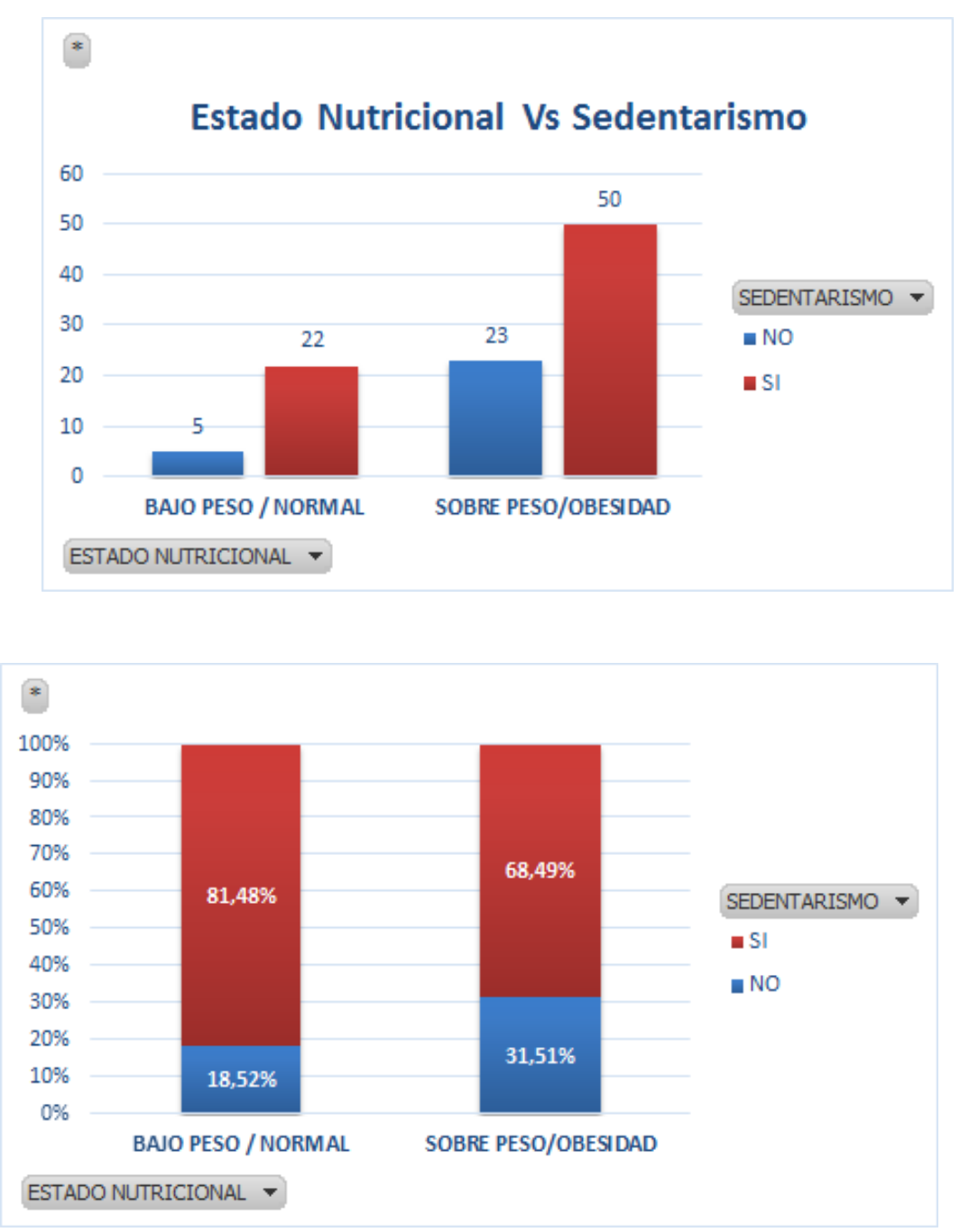

En la población total del personal administrativo de la UNEMI se identifican valores de sedentarismo que afectan al $72 \%$ de los integrantes, siendo este valor bastante alto para el área administrativa, conllevando esto a que en múltiples ocasiones los integrantes de la UNEMI no puedan desarrollar sus labores de manera eficiente o a cabalidad ocasionando múltiples faltas en el desarrollo de la jornada laboral, llegando a ser al final estos problemas de competencia para el estado que tiene que velar por el bienestar de todos sus ciudadanos.

\section{Discusión.}


A través del proceso de investigación se determinaron los principales resultados del trabajo al identificar el estado nutricional de los miembros del personal administrativo de la UNEMI, y su relación con respecto al género de los individuos, así como los factores de riesgo a los que están sometidos los participantes como: el grupo etario, educación alimentaria, raciones de los alimentos, ingesta de comida procesada, y presencia de sedentarismo, las mismas que se asocian negativamente en el proceso de nutrición, y, un proceso de malnutrición incide negativamente en las enfermedades tornándose un ciclo vicioso en la relación, lo que afecta a los integrantes tanto en su desarrollo como en su vida diaria.

Con el instrumento aplicado se logró identificar 58 son femeninos y 42 son masculinos de los cuales se realizó un contraste con el estudio realizado en la ciudad de lima sobre los posibles factores de riesgos que determinaron la obesidad y malnutrición de una institución de educación superior en la misma ciudad, acertando en los diversos tipo de investigación y de recolección de datos y confirmando con ellos las variables comunes y asociándolos con enfermedades cardiovasculares en personas de grupo etario de 40-50 años. (Rosas, Lama, Llanos, \& Dunstan, 2014)

Además, el resultado obtenido por el factor de riesgo como el sexo de los colaboradores, se analizó con un estudio realizado en la habana Cuba, por el cual obteniendo una mayor afectación de la malnutrición en personas del sexo femenino de edades de 20 a 59 años afectadas con los valores de $91,78 \%$ con edades de 25 a 45 años de edad con afectaciones al sobrepeso y obesidad presente en la investigación realizada. (Berdasco Gómez \& Romero del Sol, 2014)

Se encontró un estudio que analizaba la alimentación de los empleados con alto contenido de azucares y grasas, carnes y cerdos, de los cuales repercuto en grados de obesidad de los empleados, 
en base al estudio que se realizó los individuos de análisis aceptaron la ingesta inadecuada de alimentos procesados, afectando su estado nutricional y funcional en las labores diarias. Ambos estudios identificaron los malos hábitos alimentos de las personas de acuerdo a la ingesta y en el horario que los consumían y se sumaba que no tenía una vida activa durante todo el día, puntos fundamentales para la determinación de afectación de los factores de riesgos prevalentes sobre la obesidad afectada. (Salinas, Lera, González, Villalobos, \& Vio, 2014)

Los factores de riesgo como la raciones alimenticias, tiempo de alimentos y lugar donde se los come son de gran ayuda para determinar los índice de obesidad en las personas, esto se ve reflejado en el estado nutricional de cada uno ya que no existe una educación y concienciación alimentaria sobre los correctos hábitos alimenticios imposibilitando a las personas la recuperación de un estado óptimo y generando enfermedades patológicas asociadas a la malnutrición. (Berdasco Gómez \& Romero del Sol, 2014)

Por último la sociedad española de nutrición declara que los factores de riesgos nutricionales en las personas que laboran en empresas se indican una gran prevalencia en el sexo femenino mostrando una vulnerabilidad en la salud, asociados a la falta de actividad física y por ende un sedentarismo notorio, en cambio en el sexo masculino, se mostró que los factores que influyen en la obesidad presentan gran parte por la ingesta de alimentos inadecuada generando en ellos problemas de hipertensión y sobre peso. (Salinas, Lera, González, Villalobos, \& Vio, 2014)

\section{Bibliografía.}

Berdasco Gómez, A., \& Romero del Sol, J. M. (2014). Circunferencia del brazo como evaluadora del Estado nutricional del adulto. Revista Cubana Aliment Nutr, 12(2), 86-90.

Montejano Lozoya, R., Ferrer Diego, R., Gonzalo, C., Martínez-Alzamora, N., Sanjuan Quiles, Á., \& Ferrer Ferrándiz, E. (2014). Factores asociados al riesgo nutricional en adultos mayores autónomos no institucionalizados. Nutrición Hospitalaria, 30(4). 
Yazmin de las Mercedes Granda-Barba; Amalin M. Chávez-Gallegos; Elvia P. Aspiazu-Miranda

Organización Mundial de la Salud. (27 de 0CTUBRE de 2012). Controlar los factores de riesgo para la salud podría prevenir millones de muertes. Ginebra: OMS.

Rosas, A., Lama, F., Llanos, Z., \& Dunstan, Y. (2014). Prevalencia de obesidad e hipercolesterolemia en trabajadores de una institución estatal de Lima Perú. Revista Perú Medicina Exp. Salud Pública.

Salinas, J., Lera, L., González, C., Villalobos, E., \& Vio, F. (jul de 2014). Estilo de vida, alimentación y estado nutricional en trabajadores de la construcción de la región Metropolitana de Chile. Revista médica de Chile, 142(7).

Vásquez-Garibay, E., \& Romero Velarde, E. (2005). Valoración del estado de nutrición. En E. RomeroVelarde, SAM Nutrición Pediátrica (págs. 111-124). México: Academia Mexicana de Pediatría.

Vásquez-Garibay, E., Ávila-Alonso, E., Contreras-Ramos, T., Cuellar-Espinosa, L., \& Romero-Velarde, E. (2007). Factores de riesgo asociados al estado nutricio en lactantes que son atendidos en guarderías. Boletín médico del Hospital Infantil de México, 64(1). 